
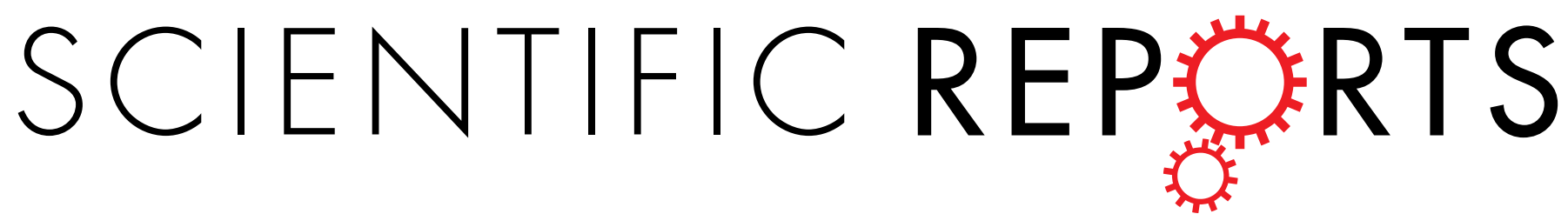

\title{
OPEN Universal diffusion-limited injection and the hook effect in organic thin-film transistors
}

Received: 02 April 2016

Accepted: 24 June 2016

Published: 21 July 2016

\author{
Chuan Liư ${ }^{1}$, Gunel Huseynova², Yong Xu2 , Dang Xuan Long², Won-Tae Park², Xuying Liư ${ }^{3}$, \\ Takeo Minari ${ }^{3} \&$ Yong-Young Noh ${ }^{2}$
}

The general form of interfacial contact resistance was derived for organic thin-film transistors (OTFTs) covering various injection mechanisms. Devices with a broad range of materials for contacts, semiconductors, and dielectrics were investigated and the charge injections in staggered OTFTs was found to universally follow the proposed form in the diffusion-limited case, which is signified by the mobility-dependent injection at the metal-semiconductor interfaces. Hence, real ohmic contact can hardly ever be achieved in OTFTs with low carrier concentrations and mobility, and the injection mechanisms include thermionic emission, diffusion, and surface recombination. The non-ohmic injection in OTFTs is manifested by the generally observed hook shape of the output conductance as a function of the drain field. The combined theoretical and experimental results show that interfacial contact resistance generally decreases with carrier mobility, and the injection current is probably determined by the surface recombination rate, which can be promoted by bulk-doping, contact modifications with charge injection layers and dopant layers, and dielectric engineering with high- $k$ dielectric materials.

The charge injection at the metal-semiconductor interface is one of the main limiting factors of many non-silicon-based electronic devices, especially those with organic semiconductors ${ }^{1}$. The energy barriers and/or interface states at the heterojunction contacts considerably consume voltage drop ${ }^{2,3}$, generate Joule heating ${ }^{4}$, and decrease injection efficiency. Especially, a large contact resistance seriously suppresses the performance of thin-film transistors (TFTs) used for the back panel of displays ${ }^{5}$, sensors, memories, and other facile electronics, which call for a low driving voltage and a high on-off ratio ${ }^{6}$. Such limitations raise the demand for ohmic-contact injection $^{7,8}$, which has not been achieved in most organic TFTs (OTFTs), or organic field-effect transistors (OFETs). To solve the problem, we need to understand what procedure carriers experience at the injection, as well as how contact resistance is determined by the materials, interfaces, device, and operational conditions.

Injection barriers generally exist at the contacts of OTFTs and are caused by mismatched work functions of metals with respect to the injection levels in organic semiconductors ${ }^{9}$, or interfacial states with charge-transfer and dipole moments ${ }^{1,10}$. The injection barrier leads to a depletion region ${ }^{11}$ where carriers are depleted and need to diffuse through the region after a number of collisions. The depletion width $\left(W_{\mathrm{d}}\right)$ can be estimated by knowing the effective barrier height $(\sim 0.5 \mathrm{eV})$, band-gap $(\sim 2 \mathrm{eV})$, and Fermi-level of organic semiconductors (OSCs) (near the mid-gap). Using common parameters of intrinsic OSCs in the literature, $W_{\mathrm{d}}$ is estimated to be over $1 \mu \mathrm{m}$ in OTFTs $^{12}$. This value is much larger than the mean free path $\left(l_{\text {free }}\right)$ of a carrier in OSCs that is only at the order of intermolecular distance (several $\AA$ to $\mathrm{nm}$ ) owing to structural disorders and localized polarons ${ }^{13,14}$. Moreover, the estimated $W_{\mathrm{d}}$ is much larger than the usual thickness of active layers $(10 \mathrm{~nm}<t<100 \mathrm{~nm})$, pointing to the possibility of diffusion-limited injection. However, there is still a lack of direct evidence in OTFTs that charge injection is diffusion-limited, as well as a need for quantitative studies to understand how this limitation affects charge injection and contact resistance in OTFTs. 


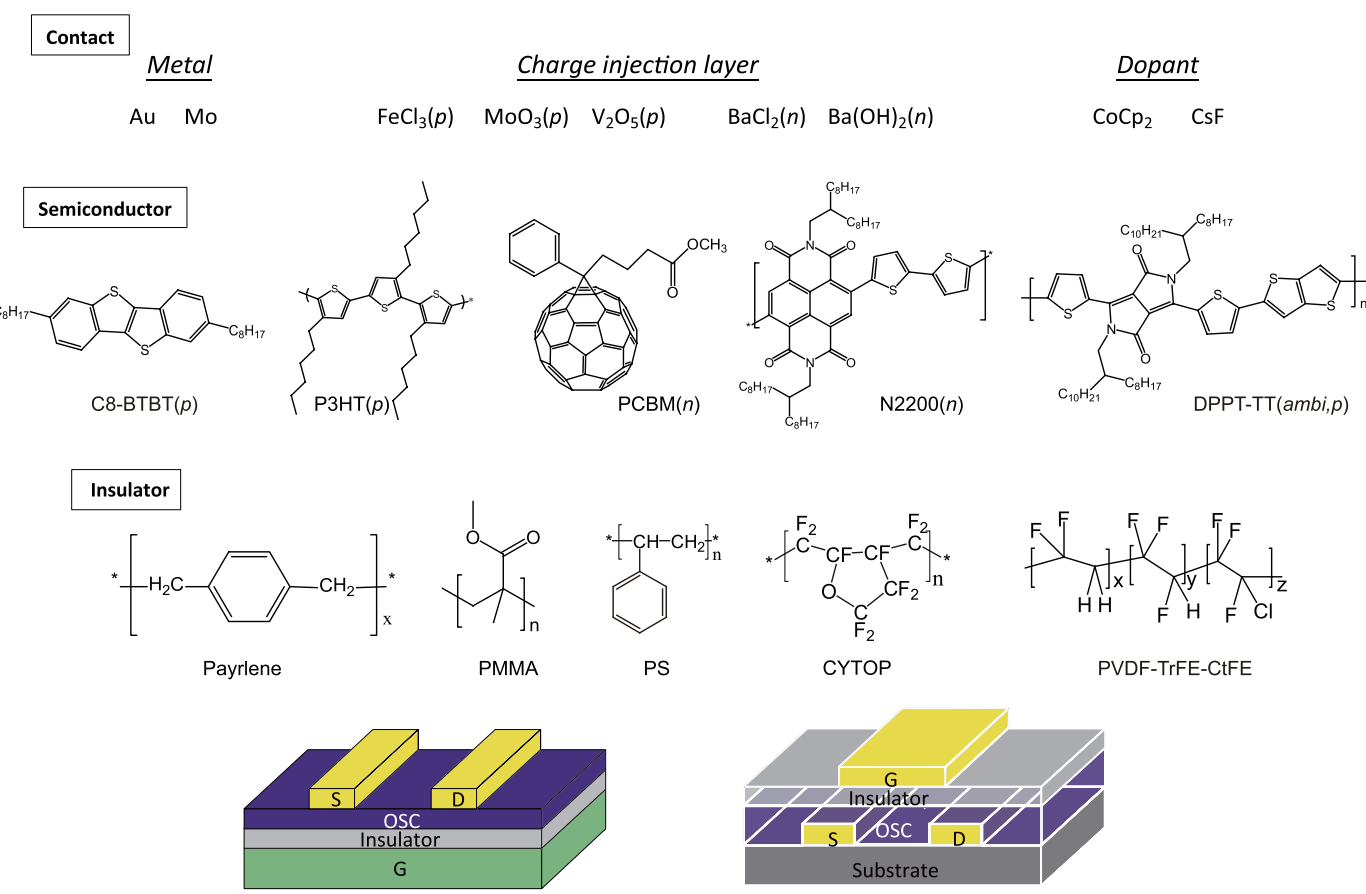

Figure 1. Materials for contact, semiconductors, and dielectric layers, and device structures used in this study.

In this work, we analyzed different injection mechanisms and derived a general form of interfacial contact resistance $\left(R_{c, \text { int }}\right)$, which is expressed as a function of the effective injection barrier, carrier mobility, and drain voltage in staggered OTFTs. We propose that, in OTFTs with non-ohmic injection, a hook shape would appear in conductance-drain voltage $\left(G-V_{\mathrm{d}}\right)$ relations, and we experimentally observed such an effect in OTFTs made with a broad range of materials. The hook effect indicates that as the drain field increases, OTFTs experience a transition from the injection-limited to the field-effect transport regime. By analyzing various OTFTs with our developed tool, we reveal that the interfacial contact resistance (readily excluding the bulk resistance) is closely related to carrier mobility and the charge injection in OTFTs is universally diffusion-limited. Importantly, the values of $R_{c \text {,int }}$ followed our proposed general form in the case of surface recombination process, i.e., carriers recombine with the image charges at the metal-semiconductor interface in OTFTs. Thus, the semiconductor mobility plays a critical role in the injection, probably by determining the surface recombination velocity and rate.

\section{Results}

Materials and structures of OTFTs. To investigate the contact properties of OTFTs, we fabricated a wide range of OTFTs covering a variety of materials. The basic structures are bottom-gate/top-contact (BG/ TC) and top-gate/bottom-contact (TG/BC) with gold source and drain electrodes, unless stated otherwise (see Fig. 1). For organic semiconductors, we used: (1) p-type small molecule dioctylbenzothienobenzothiophene (C8-BTBT) and polymer poly(3-hexylthiophene) (P3HT); (2) n-type small molecule [6,6]-phenyl-C61-butyric acid methyl ester (PCBM, small molecule) and polymer poly [[N, $\mathrm{N}^{\prime}$-bis(2-octyldodecyl)-1,4,5,8-naphthalene-dicarboximide-2,6-diyl]-alt-5,5'-(2,2'-bithiophene)] [P(NDI2OD-T2) or N2200]; and (3) ambipolar poly[[2,5-bis(2-octyldodecyl)-2,3,5,6-tetrahydro-3,6-dioxopyrrolo[3,4-c]pyrrole-1,4-diyl]-alt-[[2,2'-(2,5-thiophene) bis-thieno(3,2-b)thiophene]-5,5'-diyl]] (DPPT-TT). The materials include the commonly used thieno-thiophene-based, thiophene-based, $\mathrm{C}_{60}$-based, perylene-based, and diketopyrrolopyrrole (DPP)-based organic semiconductors. To modify the contacts, different types of contact engineering methods and materials were used, including selectively inserting a doping layer $\left(\mathrm{FeCl}_{3}\right)$, inserting a charge injection layer $\left[\mathrm{MoO}_{3}\right.$, $\left.\mathrm{V}_{2} \mathrm{O}_{5}, \mathrm{BaCl}_{2}, \mathrm{Ba}(\mathrm{OH})_{2}\right]$, and doping the bulk semiconductor with dopants (CoCp $\left.\mathrm{p}_{2}, \mathrm{CsF}\right)$. For the dielectrics, we selected commonly used low- $k$ polymer dielectrics [parylene, poly(methyl methacrylate) (PMMA), polystyrene (PS)], and a high- $k$ dielectric poly(vinylidene fluoride-ter-trifluoroethylene-ter-chlorotrifluoroethylene) [P(VDFTrFE-CtFE)]. The semiconducting and dielectric layers of the devices were all solution-processed by spin coating, except the device with the C8-BTBT layer, which was vacuum evaporated, and the device with the parylene layer, which was chemical-vapor deposited.

Interfacial contact resistance in OTFTs. The injection barriers cause the injection current to increase non-linearly with the applied voltage $V_{\mathrm{a}}$ because injections may occur with the mechanisms illustrated in Fig. 2. When depletion width $W_{\mathrm{d}}$ is smaller than the mean free path $l_{\text {free, }}$ only thermionic emission (denoted as "e") or tunneling processes (denoted as " $\mathrm{t}$ ") dominate (Fig. 2a,b) and the current follows ${ }^{15}$ : 

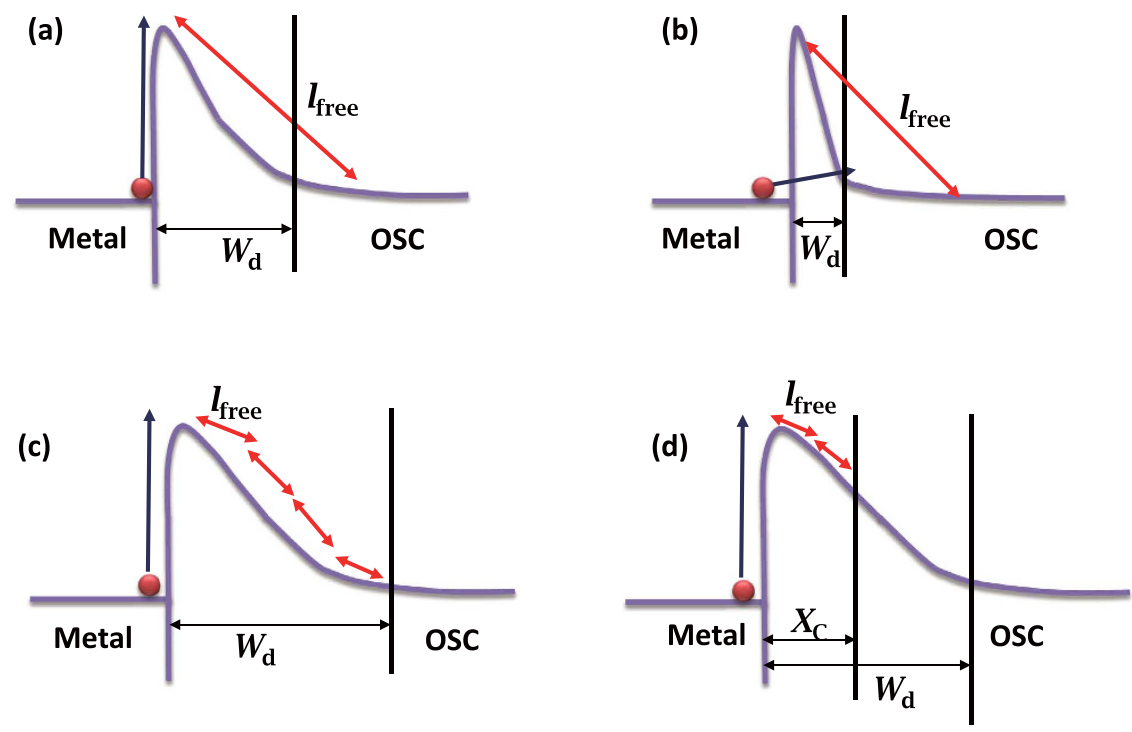

Figure 2. Schematic representations of charge injection mechanisms (take electron injections for an example): (a) thermionic emission; (b) tunneling; (c) thermionic diffusion; and (d) thermionic emission with surface recombination. The injections via thermal activation or tunneling are indicated with dark blue arrows and the mean free paths are indicated with red arrows. The characteristic lengths mentioned in the main text are also illustrated.

$$
\begin{gathered}
I_{\mathrm{e}}=A^{*} T^{2} S \exp \left(-\frac{q \varphi_{\text {eff }}}{k T}\right) \exp \left(\frac{q V_{\mathrm{a}}}{k T}\right) \\
I_{\mathrm{t}} \propto \exp \left(-\frac{q \varphi_{\text {eff }}}{E_{00}}\right) \exp \left(\frac{q V_{\mathrm{a}}}{k T}\right)
\end{gathered}
$$

Here $A *$ is the Richardson constant, $T$ is the temperature, $k$ is the Boltzmann constant, $S$ is the injection area, $\varphi_{\text {eff }}$ is the effective Schottky barrier height that takes into account the density of states distributions ${ }^{16}, E_{00}$ is related to doping concentrations in semiconductors, and $V_{\mathrm{a}}$ is the applied voltage across the metal-semiconductor junction, which is assumed to follow drain voltage $V_{\mathrm{d}}$ in OTFT as $V_{\mathrm{a}} \propto\left(V_{\mathrm{d}}\right)^{\alpha}$. The image-force-induced lowering of the injection barrier (Schottky effect) is not considered here for simplicity and the forms of $R_{c, \text { int }}$ are:

$$
\begin{gathered}
\left(R_{\mathrm{c}, \text { int }}\right)_{\mathrm{e}}=\frac{V_{\mathrm{a}}}{I} \propto \frac{1}{A^{*} T^{2} S}\left(V_{\mathrm{d}}\right)^{\alpha} \exp \left[-B_{1}\left(V_{\mathrm{d}}\right)^{\alpha}\right] \exp \left(\frac{q \varphi_{\text {eff }}}{k T}\right) \\
\left(R_{\mathrm{c}, \text { int }}\right)_{\mathrm{t}} \propto\left(V_{\mathrm{d}}\right)^{\alpha} \exp \left[-B_{2}\left(V_{\mathrm{d}}\right)^{\alpha}\right] \exp \left(\frac{\varphi_{\text {eff }}}{E_{00}}\right)
\end{gathered}
$$

Here the parameters $B$ are the constant of $V_{\mathrm{d}}$ (this also applies to the following equations).

However, when depletion width $W_{\mathrm{d}}$ is much larger than the mean free path $l_{\text {free }}$, which is usually the case in OTFTs, carriers experience many collisions across the depletion region (Fig. 2c) and the injection becomes diffusion-limited. The thermionic emission-diffusion model (denoted as "ed") predicts the injection current as ${ }^{15}$ :

$$
I_{\text {ed }}=q N_{\mathrm{c}} \mu_{\text {int }} \epsilon S \exp \left(-\frac{q \varphi_{\mathrm{Bn}}}{k T}\right) \exp \left(\frac{q V_{\mathrm{a}}}{k T}\right)
$$

Assuming that the interfacial electric field $\epsilon \propto\left(V_{\mathrm{d}}\right)^{\beta}$ and the interfacial mobility follows the channel mobility $\mu_{\text {int }} \propto \mu^{\gamma}, R_{\text {c,int }}$ is:

$$
\left(R_{c, \text { int }}\right)_{\text {ed }} \propto \frac{1}{q N_{\mathrm{c}} \mu^{\gamma}}\left(V_{\mathrm{d}}\right)^{\alpha-\beta} \exp \left[-B_{3}\left(V_{\mathrm{d}}\right)^{\alpha}\right] \exp \left(\frac{q \varphi_{\mathrm{eff}}}{k T}\right)
$$

Here $q$ is the elementary charge and $N_{\mathrm{c}}$ is the effective density of states in the conduction band. Especially, Emtage et al. showed that, at the metal-organic interface, carriers recombine with their image charges when thermal energy $k T$ reaches the carrier-image binding energy ${ }^{17,18}$, and the recombination can occur with unipolar carriers. The critical distance for the recombination $x_{\mathrm{C}}$ for common OSCs $(\sim 5 \mathrm{~nm})^{18}$ is larger than or comparable with $l_{\text {free }}$ 


\begin{tabular}{|c|c|c|c|c|}
\hline & $R$-function & G-function & $G_{\text {dif }}$-function & $G^{*}$-function \\
\hline Key parameter & $R_{\mathrm{tot}}=\frac{V_{\mathrm{d}}}{I_{\mathrm{d}}}$ & $G=\frac{I_{\mathrm{d}}}{V_{\mathrm{d}}}$ & $G_{\mathrm{dif}}=\frac{\partial I_{\mathrm{d}}}{\partial V_{\mathrm{d}}}$ & $G^{*}=\sqrt{\frac{I_{\mathrm{d}}^{2}}{\left|V_{\mathrm{d}}\right|}\left(G-G_{\mathrm{dif}}\right)^{-1}}$ \\
\hline$\mu$ & - & $\frac{2}{C_{\mathrm{L}}}\left|\operatorname{slope}\left(G, V_{\mathrm{d}}\right)\right|$ & $\frac{1}{C_{\mathrm{L}}}\left|\operatorname{slope}\left(G_{\mathrm{dif}}, V_{\mathrm{d}}\right)\right|$ & $\frac{2}{C_{\mathrm{L}}}\left[\operatorname{slope}\left(G^{*}, V_{\mathrm{d}}\right)\right]^{2}$ \\
\hline$V_{\text {th }}$ & - & $V_{\mathrm{g}}+\frac{\operatorname{intercept}\left(G, V_{\mathrm{d}}\right)}{2 \operatorname{siope}\left(G, V_{\mathrm{d}}\right)}$ & $V_{\mathrm{g}}+\frac{\operatorname{intercept}\left(G_{\mathrm{dif}}, V_{\mathrm{d}}\right)}{\operatorname{slope}\left(G_{\mathrm{dif}}, V_{\mathrm{d}}\right)}$ & $V_{\mathrm{g}}+\frac{\text { intercept }\left(G^{*}, V_{\mathrm{d}}\right)}{2 \operatorname{slope}\left(G^{*}, V_{\mathrm{d}}\right)}$ \\
\hline$R_{\mathrm{ch}}$ & - & $\frac{1}{\mid \text { intercept }+ \text { slope } \times V_{\mathrm{d}} \mid}$ & $\frac{1}{\mid \text { intercept }+ \text { slope } \times \frac{V_{\mathrm{d}}}{2} \mid}$ & $\frac{1}{\mid G^{*} \times \text { slope } \mid}$ \\
\hline$R_{\mathrm{c}}$ & $\sim R_{\text {tot }}$ & $\left(R_{\mathrm{tot}}-R_{\mathrm{ch}}\right)$ & $\left(R_{\mathrm{tot}}-R_{\mathrm{ch}}\right)$ & $\left(R_{\mathrm{tot}}-R_{\mathrm{ch}}\right)$ \\
\hline
\end{tabular}

Table 1. The derived functions to estimate $\boldsymbol{R}_{\mathrm{c}, \text { int }}$ from output characteristics. The expressions are applicable for both $p$ - and $n$-type devices (note that $V_{\mathrm{d}}$ and $I_{\mathrm{d}}$ have the same sign). The extrapolated parameters are expressed in "slope (y, x)" and "intercept $(\mathrm{y}, \mathrm{x})$ ". In calculations, we defined $G_{\mathrm{dif}}$ as $G_{\mathrm{dif}}(n)=\left[I_{\mathrm{d}}(n)-I_{\mathrm{d}}(n-1)\right] /$ $\left[V_{\mathrm{d}}(n)-V_{\mathrm{d}}(n-1)\right]$, where $n$ is the number of measured data. Here it is assumed that $R_{\mathrm{c}, \text { bulk }}$ can be ignored and, when it is not ignorable, it is included in the extracted $R_{\mathrm{ch}}$.

(several $\AA$ to a few nm) (Fig. 2d). The recombination current is opposite to the thermionic injected current and Scott et al. calculated the detailed balance between the two to derive the net injection current ${ }^{18}$ :

$$
I_{\mathrm{r}}=4 S \psi^{2} N_{0} q \mu_{\text {int }} \epsilon \exp \left(-q \varphi_{\text {eff }} / k T\right) \exp \left[\left(\frac{q r_{\mathrm{C}}}{k T} \epsilon\right)^{1 / 2}\right]
$$

Here the subscript " $r$ " denotes surface recombination, $\psi$ is a function slowly varying with electric field $\epsilon, N_{0}$ is the total hopping sites in the organic semiconductor (similar to the above $N_{\mathrm{c}}$ in inorganic semiconductors), and $r_{\mathrm{C}}$ is the Coulomb radius $\left(r_{\mathrm{C}} \sim 4 x_{\mathrm{C}}\right)$. The mobility appears because it determines the surface recombination velocity near the interface. This form was supported by experiments in a metal-organic semiconductor diode by Shen et al. ${ }^{19}$ and so $R_{\mathrm{c}, \text { int }}$ should follow:

$$
\left(R_{\mathrm{c}, \text { int }}\right)_{\mathrm{r}} \propto \frac{1}{4 S \psi^{2} N_{0} q \mu^{\gamma}}\left(V_{\mathrm{d}}\right)^{\alpha-\beta} \exp \left[-B_{4}\left(V_{\mathrm{d}}\right)^{\frac{\beta}{2}}\right] \exp \left(\frac{q \varphi_{\text {eff }}}{k T}\right)
$$

From the above models, we can summarize Eqs 2, 4, 6, and 8 to give a general form as:

$$
R_{c, \text { int }} \propto \mu^{-\gamma} \times\left(V_{\mathrm{d}}\right)^{a} \exp \left[-B\left(V_{\mathrm{d}}\right)^{b}\right] \times \exp \left(\frac{\varphi_{\mathrm{eff}}}{\varphi_{0}}\right)
$$

Here $\varphi_{0}$ ideally equals $\frac{k T}{q}$ except in the tunneling model where $\varphi_{0}=E_{00}$, and the parameter $B$ generally decreases with thermal energy $\frac{k T}{q}$. In the non-diffusion-limited cases, including thermionic emission and the tunneling models, $\gamma=0$ and $a=b$; in the diffusion-limited case, including thermionic emission-diffusion and the surface recombination models, $\gamma>0$ and usually $a \neq b$. We expect a diffusion-limited injection in OTFTs because $l_{\text {free }}<<W_{\mathrm{d}}$ and it is probably accompanied by a surface recombination process as $l_{\text {free }} \leq x_{\mathrm{C}}$.

Extraction of interfacial contact resistance. In the following we will examine if $R_{c, \text { int }}$ in OTFTs generally follows Eq. 9, whether charge injection in OTFTs is diffusion-limited, and, if so, to what extent the charge injection is limited by diffusion. To extract $R_{\mathrm{c}, \text { int }}$, we have derived a series of functions using the $I_{\mathrm{d}}-V_{\mathrm{d}}$ relations as listed in Table 1, because conventional extraction methods (e.g., transfer-length method, TLM) ${ }^{20}$ are not applicable as they cannot exclude the bulk injection resistance $\left(R_{c, \text { bulk }}\right)$. Among these functions, the $G$-function has been introduced $^{21}$ and other functions are derived in the supplementary information (SI, part 1). The key point is that as $V_{\mathrm{d}}$ increases, $R_{c, \text { int }}$ will dramatically decrease according to Eq. 9, but $R_{\mathrm{c}, \text { bulk }}$ and $R_{\mathrm{ch}}$ will gradually increase ${ }^{21}$; then, the conductance of the OTFT increases first and then starts to decrease at a critical drain voltage $V_{\mathrm{d}}$. Thus, for both static output conductance $G=\frac{I_{\mathrm{d}}}{V_{\mathrm{d}}}$ and dynamic output conductance $G_{\mathrm{dif}}=\frac{\partial I_{\mathrm{d}}}{\partial V_{\mathrm{d}}}$, a hook shape appears near $V_{\mathrm{d}}$ ' in the $G-V_{\mathrm{d}}$ and $G_{\mathrm{dif}}-V_{\mathrm{d}}$ relation (referred to as the "hook effect" in the following).

The hook effect and the application to extract $R_{c, \text { int }}$ are shown in Fig. 3, which depicts numerical simulations on the charge injection in OTFTs. To cover general injection behaviors, we simulated $R_{\mathrm{c} \text {,int }}$ in the forms of $\exp \left(-\frac{V_{\mathrm{d}}}{P_{1}}\right)\left(\right.$ referred to as “Exp-1"), $\left(V_{\mathrm{d}}\right)^{P_{2}} \exp \left[-\left(V_{\mathrm{d}}\right)^{P_{2}}\right]$ (“Exp-2"), $\left(V_{\mathrm{d}}\right)^{P_{3}}$ ("Power"), or a constant $P_{4}$ (“Const.") (see simulation details in experimental and SI, Part 2). The simulated $I_{\mathrm{d}}-V_{\mathrm{d}}$ curves are depicted in Fig. $3 \mathrm{a}$ and the extracted $G, G_{\mathrm{dif}}$, and $G^{*}$ are shown in Fig. 3b-d. Clearly, the hook effect appears in non-linear injections but does not appear in the linear injection (i.e., $R_{\mathrm{c}}=0$, dashed lines). In Fig. $3 \mathrm{e}-\mathrm{h}, R_{\mathrm{c}, \text { int }}$ is extracted (in lines) by Table 1 and compared with the set values $R_{c, \text { set }}$ in the simulations (open squares). $G$ - and $G_{\text {dif }}$ functions give accurate estimations when $R_{\mathrm{c} \text {,int }}$ is strongly $V_{\mathrm{d}}$-dependent, while the $G^{*}$-function does so when $R_{\mathrm{c}, \text { int }}$ is $V_{\mathrm{d}}$-independent. For comparison, values of $R_{\mathrm{ch}}$ are depicted as dashed curves and they crosses the $R_{\mathrm{c}, \text { set }}$ curves at the critical $V_{\mathrm{d}}$. Furthermore, the $G$ - and $G_{\text {dif }}$-functions were examined using commercial TFT simulation tools (Silvaco Atlas) with Schottky 

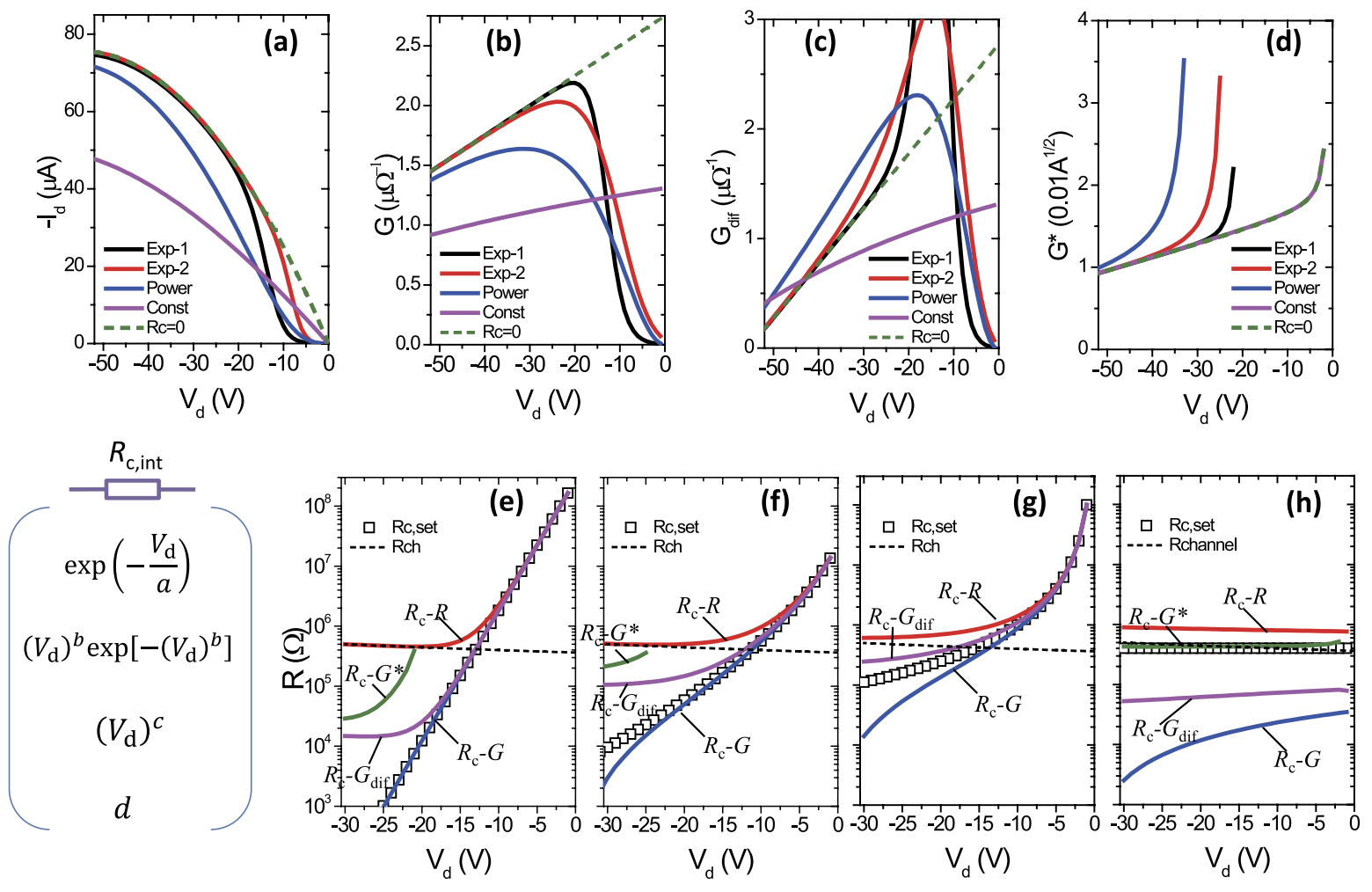

Figure 3. (a) The simulated $I_{\mathrm{d}}-V_{\mathrm{d}}$ characteristics for various contact properties. The extracted static output conductance $G$, dynamic output conductance $G_{\mathrm{dif}}$, and $G^{*}$ are depicted in (b-d), respectively. Comparison between the set values (open squares) and calculated values (lines) of $R_{c, \text { int }}$ for various contact properties: (e) "Exp-1", (f) "Exp-2", (g) "Power", and (h) "Constant". The set values of $R_{\mathrm{ch}}$ (dashed lines) are also shown for reference.

barriers as presented in the SI (Part 3), which proves good accuracy. Importantly, the extracted resistance readily excludes the main part of the bulk resistance $\left(R_{\mathrm{c}, \mathrm{bulk}}\right)$, and therefore is mainly the interfacial contact resistance $\left(R_{c, \text { int }}\right)$, as discussed in the SI (part 4). In the following, we apply the method to analyze the experimental results of various OTFTs.

Experimental results of OTFTs. The measurement and investigation of output characteristics of all OTFTs is shown in Fig. 4 ( $n$-type) and Fig. 5 ( $p$-type), and the results are summarized in Table 2. In the analysis, we intentionally skip the data next to $V_{\mathrm{d}}=0 \mathrm{~V}$ to reduce the error induced by $V_{\mathrm{d}}$-dependent mobility, because the above methods assume that mobility is weakly dependent on $V_{\mathrm{d}}$. In all OTFTs, we observed the universal hook effect, which is manifested as follows: $G$ and $G_{\text {dif }}$ start from zero, raise to a maximum and then decrease linearly in all the OTFTs, regardless of the fabrication process and material of contacts, semiconductors, and dielectrics. This is the first important finding of our study.

The hook effect is alleviated by using bulk dopants, inserting charge injection layers (CILs), or even by changing dielectrics. We take the $n$-type, BC/TG OTFTs based on N2200 semiconductors for the first example (see $I_{\mathrm{d}}$, $G$, and $G_{\text {dif }}$ in Fig. $\left.4 \mathrm{a}-\mathrm{d}\right)$. Both $G$ and $G_{\text {dif }}$ exhibit typical hook features followed by a linear relationship with $V_{\mathrm{d}}$ (see the dashed red lines). With organic dopant $\mathrm{CoCp}_{2}$ or inorganic dopant CsF in the bulk N2200, the OTFTs exhibit larger conductance ( $G$ and $G_{\text {dif }}$ above $2 \mu \Omega^{-1}$ ) as compared to the pristine device (below $0.6 \mu \Omega^{-1}$ ). We use $G$-function to calculate $R_{\mathrm{c}, \text { int }}$ and $R_{\mathrm{ch}}$ and quantitatively determine $V_{\mathrm{d}}^{\prime}\left(R_{\mathrm{c} \text {,int }}=R_{\mathrm{ch}}\right)$ for each type of device in Fig. $4 \mathrm{a}-\mathrm{c}$, which signifies the transition from the injection-dominated regime to transport-dominated regime. The critical $V_{\mathrm{d}}$ ' is above $15 \mathrm{~V}$ with pristine semiconductors, while $V_{\mathrm{d}}$ ' is below $6 \mathrm{~V}$ with bulk dopants. As the dopants $\mathrm{CoCp}_{2}$ and $\mathrm{CsF}$ increase the carrier concentration ${ }^{22}$, the conductivity adjacent to the contact is enhanced, the depletion region is narrowed ${ }^{12}$, and the $R_{c, \text { int }}$ is lowered by over 100 times.

Such improvements are also observed in OTFTs with CILs. In $n$-type OTFTs with PCBM (Fig. $4 \mathrm{~m}-\mathrm{x}$ ), the $\mathrm{Ba}(\mathrm{OH})_{2}$ CIL reduced $R_{c, \text { int }}$ as significantly as three orders of magnitude (values at $2 \mathrm{~V}$ ). In $p$-type OTFTs with $\mathrm{P} 3 \mathrm{HT} /$ Mo contacts (Fig. 5a-l), the solution-processed $\mathrm{MoO}_{3}$ and $\mathrm{V}_{2} \mathrm{O}_{5}$ CILs reduced $R_{\text {c,int }}$ by up to two orders of magnitude, and reduced $\left|V_{\mathrm{d}}^{\prime}\right|$ to below $-2 \mathrm{~V}$. The improvement comes from reducing the energy barrier according to spectroscopic observations ${ }^{23,24}$. In addition, CILs that are strong dopants also help in $p$-type OTFTs with C8-BTBT semiconductor (SI, part 4). The $\mathrm{FeCl}_{3} \mathrm{CIL}$ at the contact region increases free carriers and reduces the unoccupied traps, so that it reduced $R_{c, \text { int }}$ by about 10 times and reduced $V_{\mathrm{d}}$ ' to be unobservable. For reference, the extracted $R_{c, \text { int }}$ for C8-BTBT OTFTs with $\mathrm{FeCl}_{3}$ is $6.8 \mathrm{k} \Omega \cdot \mathrm{cm}\left(V_{\mathrm{d}}=-2 \mathrm{~V}\right)$ which is close to the value $(8.8 \mathrm{k} \Omega \cdot \mathrm{cm})$ extracted from $\mathrm{TLM}^{25}$, showing that our methods are reasonable estimations. 

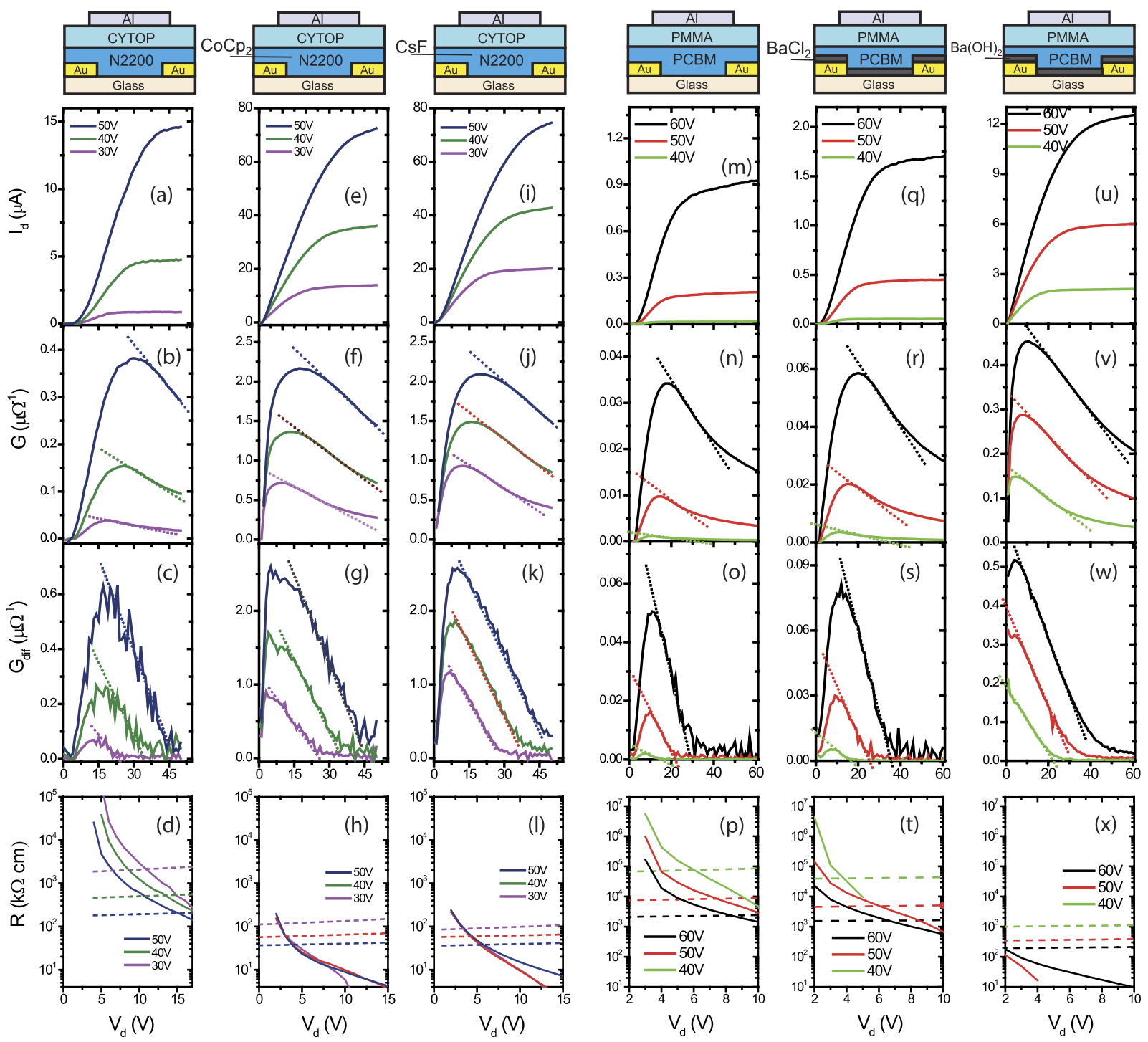

Figure 4. Charge injection of $n$-type FETs characterized by output characteristics, static output conductance $G$, dynamic output conductance $G_{\mathrm{dif}}$, and extracted interfacial contact resistance $\left(R_{\mathrm{c}, \mathrm{int}}\right)$ and channel resistance $\left(\boldsymbol{R}_{\mathrm{ch}}\right)$ for OTFTs $\mathbf{2} 200$ or PCBM semiconductors. (a-d) N2200 transistors without dopant; (e-h) N2200 transistors with $\mathrm{CoCp}_{2}$ as bulk dopants; (i-l) N2200 transistors with CsF as bulk dopants; ( $\mathbf{m}-\mathbf{p}$ ) PCBM transistors without charge injection layers (CILs); (q-t) PCBM transistors with $\mathrm{BaCl}_{2}$ as CILs; $(\mathbf{u}-\mathbf{x})$ PCBM transistors with $\mathrm{Ba}(\mathrm{OH})_{2}$ as CILs. The dotted lines in the $G$ and $G_{\text {dif }}$ figures illustrate the linear part. The dashed lines in the $R$ figures denote the extracted channel resistance $\left(R_{\mathrm{ch}}\right)$ for the corresponding voltage.

Besides bulk dopants and CILs, interesting results were also found in OTFTs with various dielectrics. In OTFTs with the DPPT-TT/Au contacts (Fig. $5 \mathrm{~m}-\mathrm{t}$ ), the devices with the low- $k$ PMMA dielectric $(k=3.5)$ exhibited $R_{\mathrm{c}, \text { int }}$ values of more than $1000 \mathrm{k} \Omega \cdot \mathrm{cm}\left(\right.$ at $\left.V_{\mathrm{d}}=-2 \mathrm{~V}\right)$ and critical $V_{\mathrm{d}}^{\prime}$ in the range of -8 to $-10 \mathrm{~V}$, while the devices with the high- $k$ PVDF-TrFE-CtFE $(k=10.4)$ featured $R_{c, \text { int }}$ values two orders lower (about $\left.20 \mathrm{k} \Omega \cdot \mathrm{cm}\right)$ and a much smaller critical $V_{\mathrm{d}}{ }^{\prime}(\sim-3 \mathrm{~V})$. The large bulk capacitance and interfacial negative dipoles of PVDF-TrFE-CtFE induced strong electrostatic coupling to increase the carrier concentration and lowered the Fermi level towards the highest occupied molecular orbital $(\mathrm{HOMO})^{26}$. The magnitude of enhancement is exceptional, considering that carrier concentration only increased about six times. The above results indicate that the hook effect generally exist as a sign of non-ohmic contact injection in OTFTs and can be used to qualitatively characterize charge injections.

Whether the charge injection is diffusion-limited or not was firstly examined by depicting the relations between $V_{\mathrm{d}}^{\prime}, R_{\mathrm{c} \text {,int }}$ ( $\left(R_{\mathrm{c}, \text { int }}\right.$ at $\left.V_{\mathrm{d}}^{\prime}\right)$, and $\mu$ of OTFTs (Fig. 6a-h). The critical electric field is calculated by $F_{\mathrm{c}}=V_{\mathrm{d}}^{\prime} / L$, and for those cases where $V_{\mathrm{d}}$ ' does not appear within the measurement window, we conservatively use the minimum $V_{\mathrm{d}}$ and the corresponding channel resistance as $R_{\mathrm{c}, \text { int }}$. The calculated mobility values are lower than those reported before calculated from the saturation regime, as the values here were extracted from Table 1, whose values are close to those calculated from the linear regime. We depict normalized $F_{\mathrm{c}}$ and $R_{\mathrm{c} \text {,int }}$ ' as a function of 

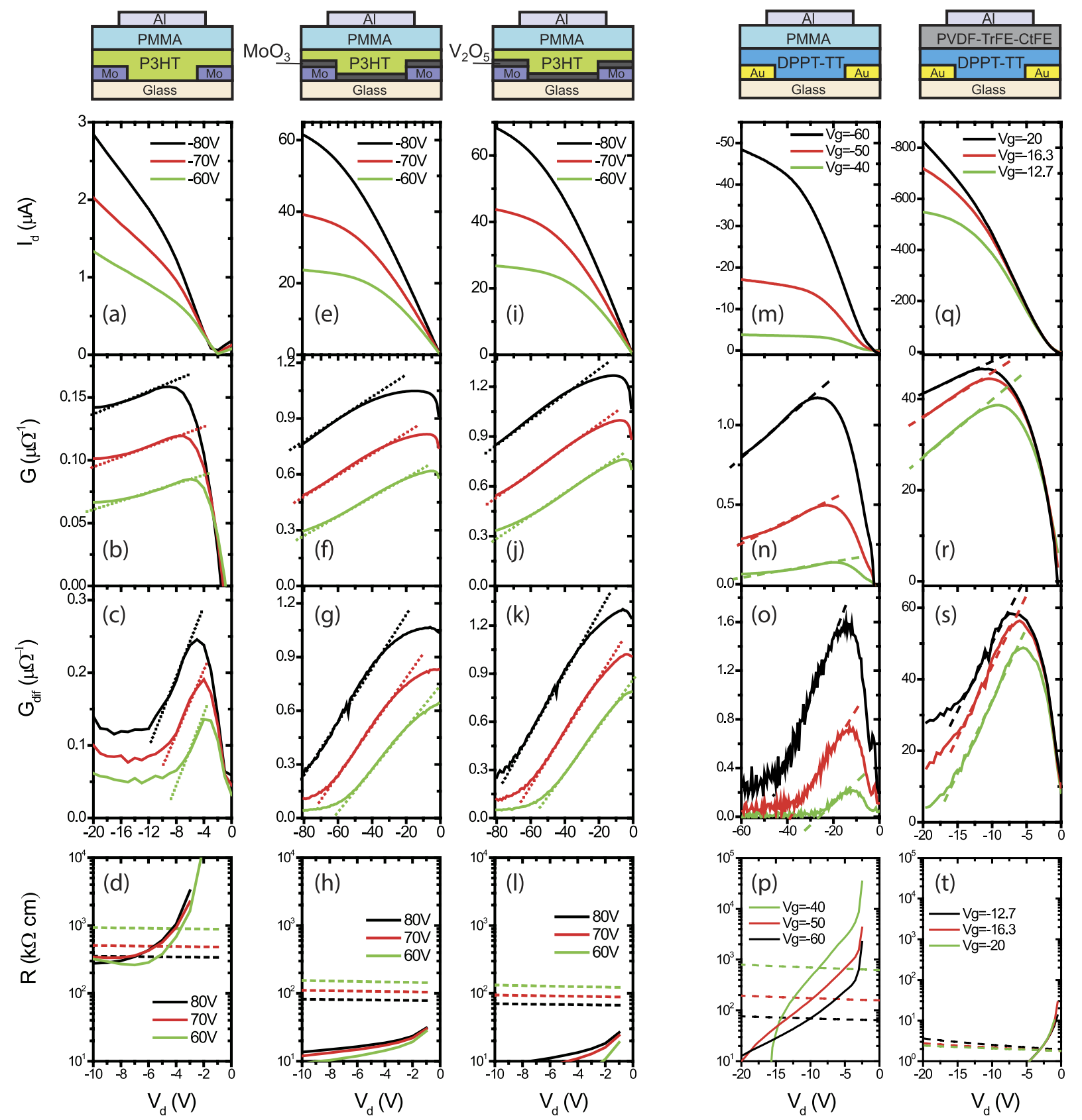

Figure 5. Charge injection of $p$-type FETs characterized by $G, G_{\mathrm{dif}}, R_{\mathrm{c} \text {,int }}$, and $\boldsymbol{R}_{\mathrm{ch}}$ for OTFTs with P3HT or DPTT-TT semiconductors. (a-d) P3HT transistors with pure Mo electrodes; (e-h) P3HT transistors with Mo electrodes covered by $\mathrm{MoO}_{3}$ CILs; (i-l) P3HT transistors with Mo electrodes covered by $\mathrm{V}_{2} \mathrm{O}_{5}$ CILs; (m-p) DPPT-TT transistors PMMA dielectric; (q-t) DPPT-TT transistors PVDF-TrFE-CtFE dielectric. The dotted lines in the $G$ and $G_{\text {dif }}$ figures illustrate the linear part. The dashed lines in the $R$ figures denote the extracted channel resistance $\left(R_{\mathrm{ch}}\right)$ for the corresponding voltage.

normalized $\mu$ for all solution-processed OTFTs (Fig. 6i,j). Here in OTFTs, it is clear that, regardless of materials, device configurations, and processing, a higher $\mu$ is related to a lower $F_{\mathrm{c}}$ and a lower $R_{\mathrm{c} \text {,int }}$. In detail, a three to four times higher $\mu$ corresponds to a three to four times lower critical field, and a $10^{2}$ to $10^{3}$ times lower $R_{c, \text { int }}$. Previous studies on the relation between mobility and injection are mainly based on diodes ${ }^{19}$. Firstly, it indicates that tuning (or optimizing) contact injection efficiency and semiconductor mobility is always synergetic, i.e., one interacts as both cause and effect with the other. Secondly, as $R_{c, \text { int }}$ ' decreases as the carrier mobility increases, the results imply that charge injections in OTFTs are diffusion-limited, as Fig. $6 \mathrm{e}-\mathrm{h}$ suggest a universal relation that $R_{\text {c,int }}$ ' decreases with $\mu$.

Diffusion-limited injection with surface recombination. In diffusion-limited injection, we especially examine Eq. 8 because the recombination process is regarded to take place in OSCs where carriers transport 


\begin{tabular}{|c|c|c|c|c|c|c|}
\hline Contact metal & Semiconductor & Dielectric & $\mathrm{C}_{\mathrm{i}}$ & $\mathbf{V}_{\mathrm{g}}$ & $\mu$ & $\mathrm{V}_{\mathrm{d}^{\prime}}$ \\
\hline \multirow{3}{*}{ Mo } & \multirow{9}{*}{ P3HT } & \multirow{9}{*}{ PMMA } & \multirow{9}{*}{$6.2 \times 10^{-9}$} & -60.0 & $2.1 \times 10^{-3}$ & -3.6 \\
\hline & & & & -70.0 & $3.7 \times 10^{-3}$ & -5.4 \\
\hline & & & & -80.0 & $4.5 \times 10^{-3}$ & -7.0 \\
\hline \multirow{3}{*}{$\mathrm{Mo} / \mathrm{MoO} 3$} & & & & -60.0 & $1.8 \times 10^{-3}$ & -2.0 \\
\hline & & & & -70.0 & $1.9 \times 10^{-2}$ & -2.0 \\
\hline & & & & -80.0 & $2.1 \times 10^{-2}$ & -2.0 \\
\hline \multirow{3}{*}{$\mathrm{Mo} / \mathrm{V} 2 \mathrm{O} 5$} & & & & -60.0 & $1.5 \times 10^{-2}$ & -2.0 \\
\hline & & & & -70.0 & $1.7 \times 10^{-2}$ & -2.0 \\
\hline & & & & -80.0 & $1.9 \times 10^{-2}$ & -2.0 \\
\hline \multirow{9}{*}{$\mathrm{Au}$} & \multirow{9}{*}{ DPTTT } & \multirow{3}{*}{ PMMA } & \multirow{3}{*}{$6.2 \times 10^{-9}$} & -40.0 & $5.6 \times 10^{-3}$ & -8.5 \\
\hline & & & & -50.0 & $2.0 \times 10^{-2}$ & -9.5 \\
\hline & & & & -60.0 & $4.2 \times 10^{-2}$ & -10.0 \\
\hline & & \multirow{6}{*}{ PVDF-TrFE-CTFE } & \multirow{3}{*}{$4.6 \times 10^{-9}$} & -60.0 & $1.4 \times 10^{-2}$ & -10.0 \\
\hline & & & & -70.0 & $2.5 \times 10^{-2}$ & -10.0 \\
\hline & & & & -80.0 & $3.7 \times 10^{-2}$ & -10.5 \\
\hline & & & & -12.7 & $3.8 \times 10^{-1}$ & -3.0 \\
\hline & & & $6.4 \times 10^{-9}$ & -16.3 & $3.2 \times 10^{-1}$ & -3.0 \\
\hline & & & & -20.0 & $2.3 \times 10^{-1}$ & -3.0 \\
\hline \multirow{3}{*}{$\mathrm{Au}$} & \multirow{9}{*}{ PCBM } & \multirow{9}{*}{ PMMA } & \multirow{9}{*}{$5.3 \times 10^{-9}$} & 40.0 & $6.3 \times 10^{-4}$ & 6.0 \\
\hline & & & & 50.0 & $3.9 \times 10^{-3}$ & 7.5 \\
\hline & & & & 60.0 & $1.1 \times 10^{-2}$ & 8.3 \\
\hline \multirow{3}{*}{$\mathrm{Au} / \mathrm{BaCl}_{2}$} & & & & 40.0 & $4.9 \times 10^{-4}$ & 3.8 \\
\hline & & & & 50.0 & $4.3 \times 10^{-3}$ & 6.0 \\
\hline & & & & 60.0 & $7.1 \times 10^{-3}$ & 6.5 \\
\hline \multirow{3}{*}{$\mathrm{Au} / \mathrm{Ba}(\mathrm{OH})_{2}$} & & & & 40.0 & $1.9 \times 10^{-2}$ & 2.0 \\
\hline & & & & 50.0 & $5.6 \times 10^{-2}$ & 2.0 \\
\hline & & & & 60.0 & $8.8 \times 10^{-2}$ & 2.0 \\
\hline \multirow{9}{*}{$\mathrm{Au}$} & \multirow{3}{*}{ N2200 } & \multirow{9}{*}{ CYTOP } & \multirow{9}{*}{$3.7 \times 10^{-9}$} & 30.0 & $4.5 \times 10^{-3}$ & 11.0 \\
\hline & & & & 40.0 & $4.3 \times 10^{-2}$ & 13.5 \\
\hline & & & & 50.0 & $5.9 \times 10^{-2}$ & 15 \\
\hline & & & & 30.0 & $9.9 \times 10^{-2}$ & 2.2 \\
\hline & N2200:CoCp2 & & & 40.0 & $6.9 \times 10^{-2}$ & 3.0 \\
\hline & & & & 50.0 & $1.8 \times 10^{-1}$ & 3.7 \\
\hline & \multirow{3}{*}{ N2200:CsF } & & & 30.0 & $1.4 \times 10^{-1}$ & 3.5 \\
\hline & & & & 40.0 & $1.8 \times 10^{-1}$ & 4.2 \\
\hline & & & & 50.0 & $1.8 \times 10^{-1}$ & 5.6 \\
\hline
\end{tabular}

Table 2. Characteristic parameters of the OTFTs investigated in this study. The uncertainty of calculated $\mu$ and $V_{\mathrm{d}}$ is estimated to be less than $30 \%$.

mainly via hopping. At the metal-organic interface, the recombination can occur between the carriers and their image charges ${ }^{18}$, where thermionically injected carriers within $x_{C}$ return to the metal surface to form the organic-to-metal current opposite to the metal-to-organic current (illustrated in Fig. 7a). This process can occur with unipolar carriers, in contrast to the recombination in the bulk of OSCs where Langevin's recombination occurs in the form of electron-hole recombination ${ }^{27,28}$, as well as trap-assisted recombination ${ }^{29}$. In this scenario, the total number of charge recombination events per unit time and per unit area, i.e., the surface recombination rate $r_{\text {rec }}$ (unit in $\mathrm{cm}^{-2} \mathrm{~s}^{-1}$ ), is determined by the carrier mobility together with the injection barrier. The surface recombination rate at zero field $r_{\text {rec }, 0}$ is (see derivation in the SI, Part 5):

$$
r_{\text {rec }, 0}=\frac{16 \pi \varepsilon \varepsilon_{0}(k T)^{2} N_{0}}{q^{3}} \mu_{\text {int }} \exp \left(-\frac{\varphi_{\text {eff }}}{k T}\right)
$$

For example, when $\varphi_{\text {eff }}=0.1 \mathrm{eV}, \mu_{\text {int }}=0.1 \mathrm{~cm}^{2} / \mathrm{Vs}, N_{0}=10^{6}$, and $\varepsilon=3.5$, the rate $r_{\text {rec }, 0}$ is $1.4 \times 10^{8} \mathrm{~cm}^{-2} \mathrm{~s}^{-1}$ at $300 \mathrm{~K}$, which is independent of $V_{\mathrm{d}}$ and well characterizes the interfacial conditions. When OTFT is operated at non-zero field, the rate $r_{\text {rec }}$ is proportional to $r_{\text {rec, }, 0}$ and will increase with $V_{\mathrm{d}}$, and the exact form of $r_{\text {rec }}$ can be found in the SI (Part 5). Importantly, $R_{\mathrm{c}, \text { int }}$ in OTFTs will decrease with increasing $r_{\text {rec }}$. The calculated $r_{\text {rec }}$ and $R_{c, \text { int }}$ as a function of $V_{\mathrm{d}}$ are shown in Fig. 7b-d and the correlation between $r_{\text {rec }}$ and $R_{c, \text { int }}$ are shown in Fig. 7e (see the SI, Part 5, for calculation details). The main features of the calculated curves are similar to the experimental results mentioned above, and hence we will use the Eq. 8 to fit the experimental data in the following. 

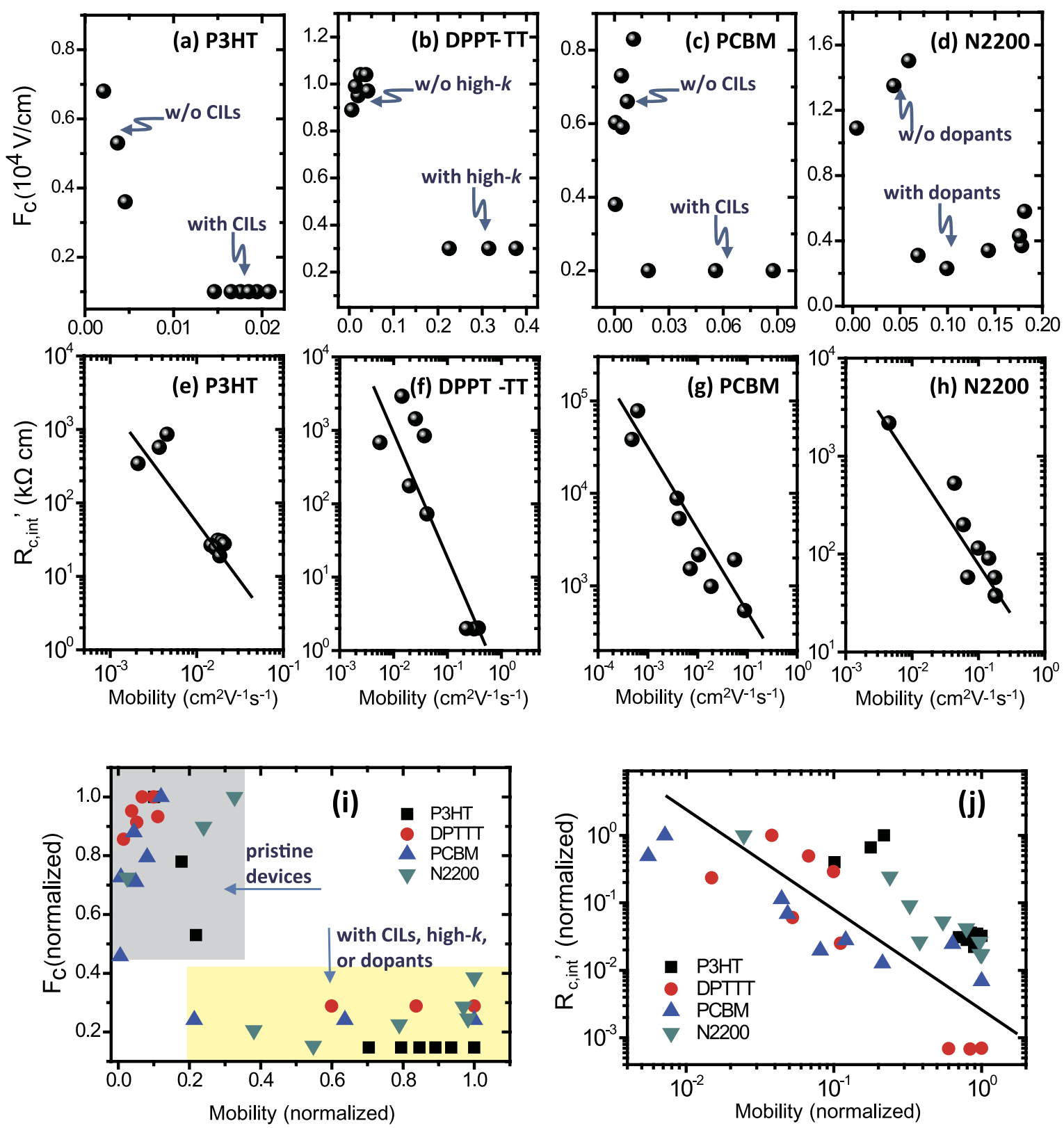

Figure 6. The critical field $F_{\mathrm{c}}(\mathbf{a}-\mathbf{d})$ and corresponding interfacial contact resistance $R_{c, \text { int }}$ (e-h) plotted against channel mobility. The values are extracted from OTFTs (shown in Figs 4 and 5) made with P3HT (a,e), DPPT-TT (b,f), PCBM (c,g), and N2200 (d,h). In (a-d), types of devices are given by texts, and the detailed structures are shown in Figs 4 and 5 . The values of $F_{\mathrm{c}}$ and $R_{\mathrm{c}, \text { int }}$ ' are normalized and plotted in the (I,j) for comparison. In (i), grey and yellow squares are used to visualize different types of devices, comparing pristine devices and those with CIL, high- $k$ dielectrics, or dopants. In $(\mathbf{e}-\mathbf{h}, \mathbf{j})$, the lines are guides for the eye.

Based on Eq. 8, for the simplicity of fitting, we assume the parameters $\alpha$ and $\beta$ to be unity and obtain (see derivation in the SI, Part 6):

$$
R_{\mathrm{c}, \text { int }} \cong A \exp \left(-B \sqrt{\left|V_{\mathrm{d}}\right|}\right)+R_{0}
$$

Here, $A \propto \frac{1}{N_{0} \mu^{\gamma}} \exp \left(\varphi_{\text {eff }} / k T\right)$ is the most important constant related to the effective Schottky barrier $\varphi_{\text {eff }}$, carrier mobility $\mu$, and number of charge hopping sites $N_{0} ; B$ is related to the local electric field and the image potential; $R_{0}$ is much smaller than the first term and it should also increase exponentially with the Schottky barrier (see the SI, Part 5). The $\mathrm{Mo} / \mathrm{V}_{2} \mathrm{O}_{5} / \mathrm{P} 3 \mathrm{HT}$ transistors (device structure is shown in Fig. 5) were examined as in those OTFTs the work-function of contact electrode was continuously tuned by changing the thickness of the $\mathrm{V}_{2} \mathrm{O}_{5}$ layers. For fitting, the values of $\varphi_{\text {eff }}$ are simply estimated by the measured injection barrier $\varphi_{\mathrm{B}}$, which is calculated by using the difference between the work function measured from $\mathrm{Mo} / \mathrm{V}_{2} \mathrm{O}_{5}$ surface and the HOMO levels of P3HT. 

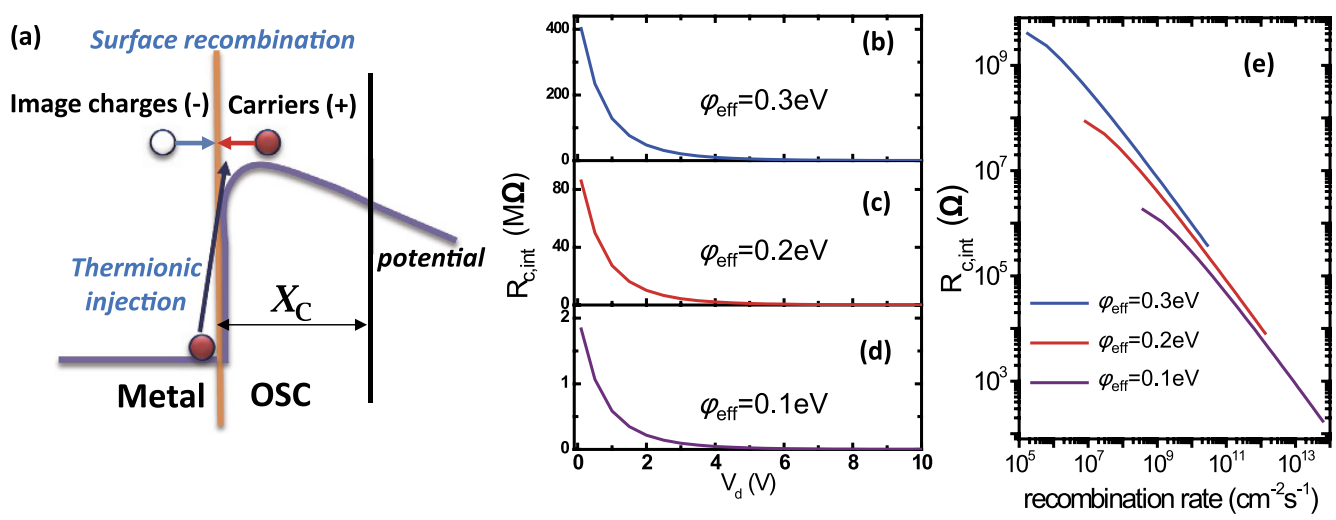

Figure 7. (a) Schematic diagram of surface recombination at the metal-organic interface (take electron current as an example). (b-d) Calculated $R_{\mathrm{c}, \text { int }}$ as a function of $V_{\mathrm{d}}$ for different injection barriers according to Eq. 8. (e) Calculated relation between $R_{\mathrm{c}, \text { int }}$ and recombination rate $r_{\text {rec }}$ in various electric field for different injection barriers.
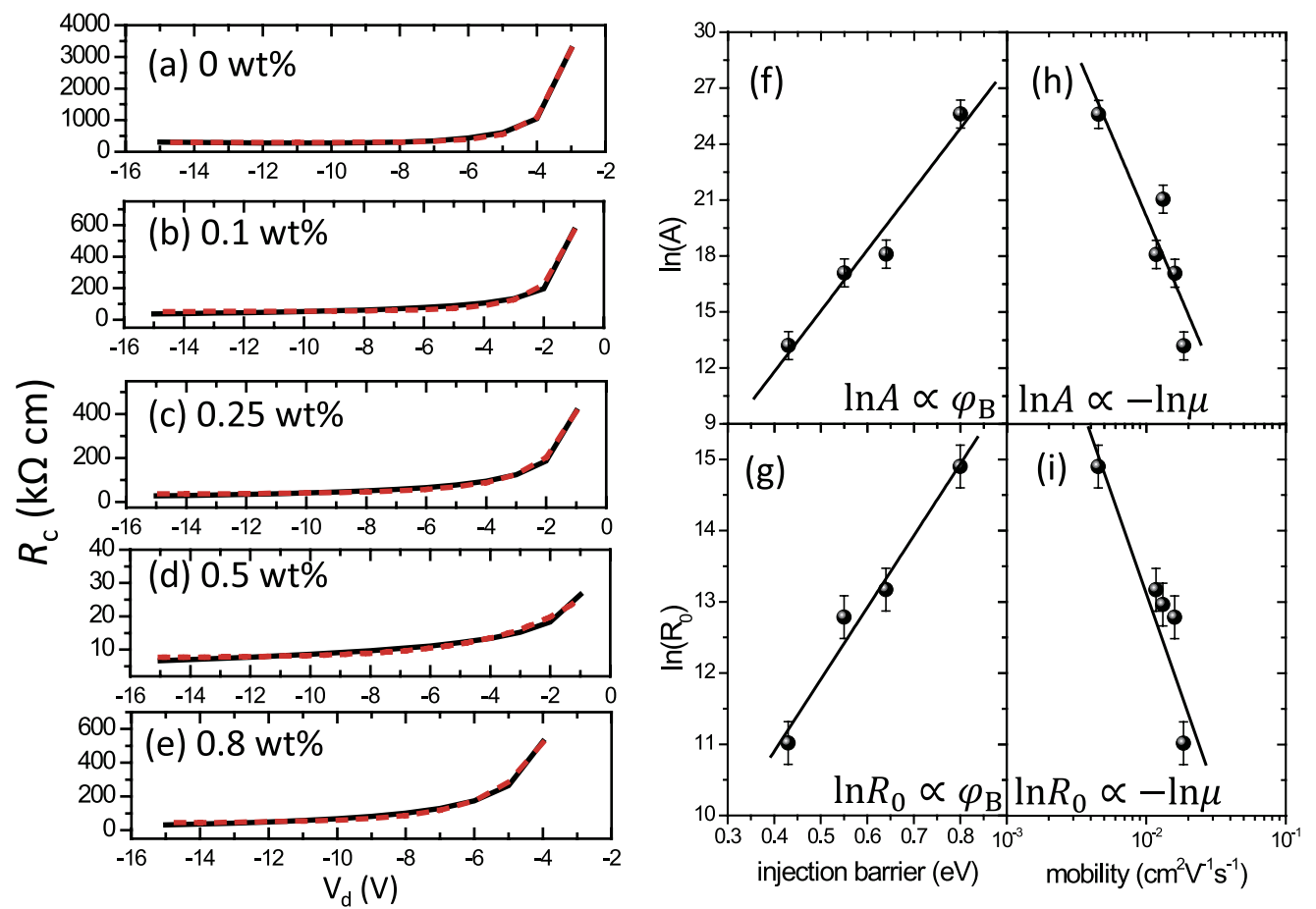

Figure 8. (a-e) Fitting the extracted values of $R_{c, \text { int }}$ to Eq. 11. The extracted $R_{c, \text { int }}$ are shown in black solid curves and the fitted curves are shown in red dashes. $(\mathbf{f}, \mathbf{g})$ Relationships between the parameter $A$ and the measured injection barrier $\varphi_{\mathrm{B}}$ and $\mu$. (h,i) Relationships between the parameter $R_{0}$ and the measured injection barrier $\varphi_{\mathrm{B}}$ and $\mu$, confirming Eqs 11-13. The measured injection barrier $\varphi_{\mathrm{B}}$ is calculated the difference between the work function measured from $\mathrm{Mo} / \mathrm{V}_{2} \mathrm{O}_{5}$ surface and the $\mathrm{HOMO}$ level of $\mathrm{P} 3 \mathrm{HT}$.

\begin{tabular}{|l|c|c|c|c|c|}
\hline & $\mathbf{0} \mathbf{w t} \%$ & $\mathbf{0 . 1} \mathbf{w t} \%$ & $\mathbf{0 . 2 5} \mathbf{w t} \%$ & $\mathbf{0 . 5} \mathbf{w t} \%$ & $\mathbf{0 . 8} \mathbf{w t} \%$ \\
\hline $\boldsymbol{A}(k \Omega \mathrm{cm})$ & $1.33 \times 10^{7}$ & $7.26 \times 10^{3}$ & $2.65 \times 10^{3}$ & $5.39 \times 10^{1}$ & $1.40 \times 10^{5}$ \\
\hline $\boldsymbol{R}_{\boldsymbol{0}}(k \Omega \mathrm{cm})$ & $2.96 \times 10^{2}$ & $5.26 \times 10^{1}$ & $3.57 \times 10^{1}$ & $6.09 \times 10^{0}$ & $4.27 \times 10^{1}$ \\
\hline $\boldsymbol{B}$ & 0.21 & 0.38 & 0.51 & 1.00 & 0.35 \\
\hline Correlation coefficient & 0.9986 & 0.9895 & 0.9941 & 0.9927 & 0.9928 \\
\hline
\end{tabular}

Table 3. Fitting results of $\boldsymbol{R}_{\mathrm{c}, \text { int }}$ to Equation 11. The device structure and fitting curves are shown in Fig. 8. The correlation coefficient $r$ for fitting are also given. 

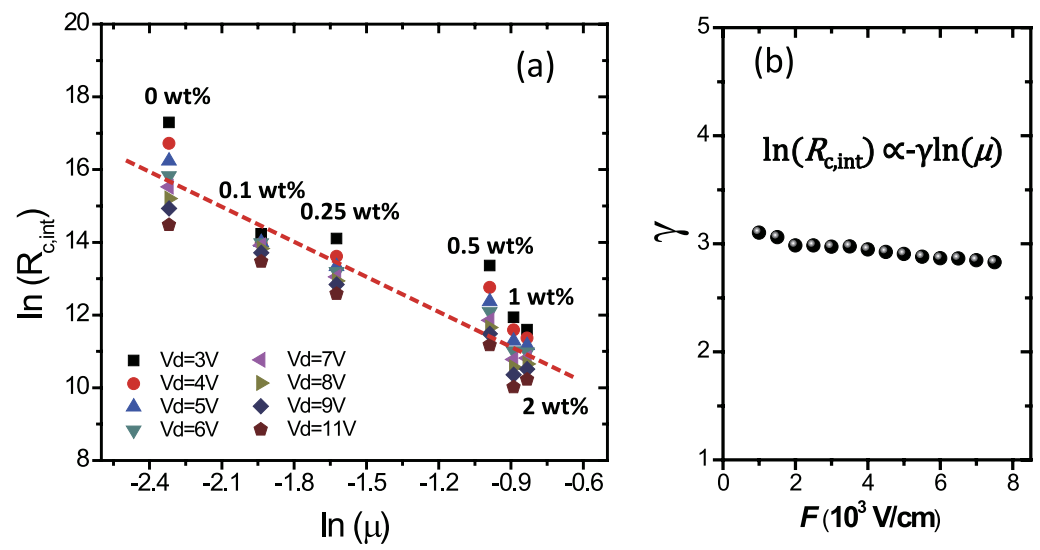

Figure 9. N2200 transistors with CsF dopants: (a) Relation between interfacial contact resistance [in terms of $\left.\ln \left(R_{c, \text { int }}\right)\right]$ and channel mobility [in terms of $\ln (\mu)$ ] for different doping concentrations (including various $V_{\mathrm{d}}$ ); the dashed line is a guide for the eye, indicating $\ln \left(R_{c, \text { int }}\right)$ decreases linearly with $\ln (\mu)$. (b) the parameter $\gamma$ for fitting $\ln \left(R_{c, \text { int }}\right) \propto-\gamma \ln (\mu)$ at different lateral fields.

Reasonable fittings of Eq. 11 were obtained for the extracted $R_{c, \text { int }}$ with different $\mathrm{V}_{2} \mathrm{O}_{5}$ layer thickness, as shown in Fig. 8a-e and Table 3. Note that when discussing injection barrier, devices spin-coated with 0 wt $\%$ to 0.5 wt $\%$ $\mathrm{V}_{2} \mathrm{O}_{5}$ solution were compared as the interlayers are thin and flat, except the $0.8 \mathrm{wt} \%$ device, which had a thick and rough insulating interlayer and the resulting extra access resistance ${ }^{23}$. In addition, according to Eq. 11, the fitting parameters should follow:

$$
\begin{aligned}
& \ln A=-C_{1} \ln \mu+C_{2} \varphi_{\text {eff }} \\
& \ln R_{0}=-C_{3} \ln \mu+C_{4} \varphi_{\text {eff }}
\end{aligned}
$$

Here $C_{1}, C_{2}, C_{3}$, and $C_{4}$ are constants for $V_{\mathrm{d}}$. Importantly, Fig. 8f-i correspond well to Eqs 12 and 13 and indicate good consistency between the theoretical derivations and the experimental results, suggesting the surface recombination scenario in OTFT injections. In fact, Eq. 10 indicates that the recombination rates can be promoted by lowering the injection barrier or/and enhancing the interfacial mobility, which has been achieved in the experimental methods mentioned above, including bulk-doping, charge injection layers, and dielectric engineering.

To further examine the general applicability of the relation between $R_{c, \text { int }}$ and carrier mobility, we analyzed the devices with n-type N2200 transistors with CsF dopants. By doping organic semiconducting layers (N2200) with dopants (CsF) in different concentrations (or weight ratios, device structure is shown in Fig. 4), the mobility of the semiconductor is tuned without changing the injection barrier and the impact of mobility on $R_{c \text {,int }}$ can be solely studied. The results are shown in Fig. 9, depicting values of $R_{c, \text { int }}$ of devices with different doping weight ratios of CsF (in wt\%). Generally, $R_{c, \text { int }}$ decreases with mobility in a power law and it is in good accordance with the theoretical prediction, which is given by the diffusion-limited injection (Eqs 6-9):

$$
\ln R_{\mathrm{c}, \text { int }} \propto-\gamma \ln \mu
$$

The power factor $\gamma$ indicates how interfacial injection is affected by channel mobility, determined by the materials and device structure. Fittings of data from N2200 transistors in Fig. 9 to the above equation are generally good as the correlation coefficients for fitting are from 0.9006 to 0.9841 . Moreover, the values of power factor $\gamma$ are around 3.0 at different drain field determined by $V_{\mathrm{d}}$ values, indicating that the assumption that mobility is almost independent of lateral electric field in the studied region. The results again confirm the above theory on the diffusion-limited injection where $R_{\mathrm{c}, \text { int }}$ follows mobility.

\section{Discussions}

Charge injection of OTFTs with various semiconductors and contact materials was investigated systematically. According to our calculations, real ohmic contact can hardly be achieved in common OTFTs with their low carrier mobilities and low carrier concentrations. Consistently, the hook effect signifying non-ohmic contact was experimentally found to be universal in OTFTs. For different non-ohmic injection mechanisms, different forms of interfacial contact resistance have been theoretically derived, which can be summarized into a general applicable form. We also developed a series of functions to analyze the interfacial contact resistances and used them to study OTFTs with a broad range of materials.

The experimental results of TFTs confirm the proposed form of interfacial contact resistance, and strongly support that OTFTs universally suffer from diffusion-limited injection, where the interfacial contact resistance generally decreases with carrier mobility. The injection process is also probably accompanied by a surface recombination process at the metal-organic interfaces. The surface recombination rate determines the injection current 
and has been promoted by bulk-doping, contact engineering with charge injection layers and dopant layers, and even dielectric engineering with high- $k$ dielectric materials. As a result, the critical voltage $\mid V_{\mathrm{d}}$ ', signifying the transition from the injection-limited to the field-effect transport regime, has also been reduced from generally over $10 \mathrm{~V}$ towards zero.

\section{Methods}

Fabrication and characterizations of OTFTs. All the fabrication and characterization of OTFTs were carried out in an $\mathrm{N}_{2}$ atmosphere unless stated otherwise.

N2200 OTFTs. A top-gate/bottom-contact (TG/BC) structure was fabricated on a glass substrate. The bottom contact electrodes were $\mathrm{Cr} / \mathrm{Au}(3 / 27 \mathrm{~nm})$. The semiconductor is N2200 or N2200 with CoCp2 and CsF doping. The $\mathrm{N} 2200$ film deposited by spin casting from the $p$-xylene solution and dried at $110^{\circ} \mathrm{C}(30 \mathrm{~nm}$ thick). The bulk dopants were mixed with $\mathrm{N} 2200$ solution in the weight ratio in $0.025 \%, 0.5 \%, 1.0 \%$, and $2.0 \%$ before spin-coating. The gate dielectric is CYTOP $\left(500 \mathrm{~nm}\right.$, deposited by spin casting and annealed at $130^{\circ} \mathrm{C}$ for 1 hour $)$. The top gate electrode is $\mathrm{Al}(50 \mathrm{~nm}$, deposited by thermal evaporation in high vacuum). The channel length is $10 \mu \mathrm{m}$ and the channel width is $1 \mathrm{~mm}$.

$P C_{61}$ BM OTFTs. The TFTs in TG/BC structure was fabricated on a glass substrate. The contact electrodes are Ni/Au $(3 / 12 \mathrm{~nm})$ deposited by thermal evaporation and patterned by photolithography. For the interlayers, $\mathrm{Ba}\left(\mathrm{OH}_{2}\right)$ and $\mathrm{Ba}\left(\mathrm{Cl}_{2}\right)$ (from Sigma-Aldrich) were dissolved in methanol $(2 \mathrm{mg} / \mathrm{ml})$ and spin-coated onto the Au patterned electrodes $(4000 \mathrm{rpm})^{24}$. The $n$-channel semiconductor $\mathrm{PC}_{61} \mathrm{BM}$ (from Nano-C) was dissolved in anhydrous chlorobenzene $(10 \mathrm{mg} / \mathrm{ml})$, spin-coated at $2000 \mathrm{rpm}$, and annealed at $110^{\circ} \mathrm{C}$ for $20 \mathrm{~min}$. The top-gate dielectric layer was deposited by spin-coating PMMA $(\mathrm{MW}=120 \mathrm{kD}$, from Sigma-Aldrich) dissolved in n-butyl acetate $(80 \mathrm{mg} / \mathrm{ml})$ followed by annealing at $80^{\circ} \mathrm{C}$ for $30 \mathrm{~min}$. The top-gate electrodes were fabricated by the thermal deposition of aluminum (Al) via metallic shadow masks ( $50 \mathrm{~nm}$ thick). The channel length is $20 \mu \mathrm{m}$ and the channel width is $1 \mathrm{~mm}$.

P3HT OTFTs. A TG/BC structure was fabricated on a glass substrate. The bottom contact electrodes were fabricated by sputtering $\mathrm{Ni} / \mathrm{Mo}(3 / 13 \mathrm{~nm})$ and conventional photolithography process to define patterns for source and drain electrodes. For the $\mathrm{MoO}_{3}$ interlayers, the ammonium molybdate $\left(\mathrm{NH}_{4}\right)_{2} \mathrm{MoO}_{4}$ in $\mathrm{H}_{2} \mathrm{O}$ solution $(0.8$ wt $\%$ of $\mathrm{MoO}_{3}$ ) was spin-coated and annealed at $150^{\circ} \mathrm{C}$ for $10 \mathrm{~min}$ in air $^{30}$. For $\mathrm{V}_{2} \mathrm{O}_{5}$ interlayers, the ammonium vanadate $\left(\left(\mathrm{NH}_{4}\right)_{3} \mathrm{VO}_{4}\right)$ solution of different concentration $\left(0.1-0.5 \mathrm{wt} \%\right.$ of $\left.\mathrm{V}_{2} \mathrm{O}_{5}\right)$ was spin-coated and then annealed at $150^{\circ} \mathrm{C}$ for $20 \mathrm{~min}$ in $\mathrm{air}^{23}$. Then semiconducting polymer rr-P3HT (from Sigma-Aldrich) dissolved in anhydrous dichlorobenzene $\left(\mathrm{DCB}, 10 \mathrm{mg} / \mathrm{ml}\right.$ ) was spin-coated and annealed at $150^{\circ} \mathrm{C}$ for $30 \mathrm{~min}$. The top-gate dielectric layer PMMA and the top Al electrodes were deposited as described above. The channel length is $10 \mu \mathrm{m}$, and channel width $W$ is $1 \mathrm{~mm}$.

C8-BTBT OTFTs. The bottom-gate electrodes are $\mathrm{Ti} / \mathrm{Au}(3 / 37 \mathrm{~nm}$, formed by vacuum evaporation), the gate dielectric was parylene $(290 \mathrm{~nm}$, deposited by chemical vapor deposition), the semiconductor was C8-BTBT ( $40 \mathrm{~nm}$ film, evaporated at $0.01 \mathrm{~nm} / \mathrm{s}$, Nippon Kayaku), and the top contact electrodes were pristine $\mathrm{Au}(50 \mathrm{~nm}) \mathrm{or}$ $\mathrm{FeCl} 3 / \mathrm{Au}(0.3 / 40 \mathrm{~nm}$, evaporated at $0.01 \mathrm{~nm} / \mathrm{s})$. The channel length is $350 \mu \mathrm{m}$ and the channel width is $1000 \mu \mathrm{m}$.

DPPT-TT OTFTs. A TG/BC structure was fabricated on a glass substrate with the bottom contact electrodes $\mathrm{Ni} / \mathrm{Au}(3 / 12 \mathrm{~nm})$ deposited as stated above. The $p$-channel semiconductor DPPT-TT was spin-coated from anhydrous chlorobenzene solution $(10 \mathrm{mg} / \mathrm{ml})$ and annealed at $200{ }^{\circ} \mathrm{C}$ for $30 \mathrm{~min}$. The gate dielectric layer is PMMA, PS, or PVDF-TrFE-CtFE (formed on top of the DPPT-TT layer by spin-coating). The capacitance per unit area is $6.2 \mathrm{nF} / \mathrm{cm}^{2}$ for PMMA, $4.6 \mathrm{nF} / \mathrm{cm}^{2}$ for PS, and $63.72 \mathrm{nF} / \mathrm{cm}^{2}$ for PVDF-TrFE-CtFE, respectively. The top gate electrode is $\mathrm{Al}(50 \mathrm{~nm}$, by thermal deposition). The channel length is $10 \mu \mathrm{m}$ and channel width $W$ is $1 \mathrm{~mm}$. In the figure for DPPT-TT $(b, f)$, the data of OTFTs using polystyrene (PS) dielectrics are also included.

Characterizations. The electrical characteristics of OTFTs were measured with an Agilent 4156A Semiconductor Parameter Analyzer in an $\mathrm{N}_{2}$ atmosphere. $L$ and $W$ are channel length and width, respectively, $\mu$ is carrier mobility in channel, $V_{\mathrm{g}}$ is gate-voltage, and $V_{\mathrm{th}}$ is the threshold voltage.

Calculation and simulation of interfacial contact resistance. Data in Fig. 2 in the main text were calculated by simulating the $I_{\mathrm{d}}-V_{\mathrm{d}}$ characteristics in the linear regime using the equation (Fig. 2):

$$
I_{\mathrm{d}}=\frac{V_{\mathrm{d}}}{R_{\text {tot }}}=\frac{V_{\mathrm{d}}}{R_{\mathrm{c} \text {,int }}+R_{\mathrm{c} \text {,bulk }}+R_{\text {channel }}}
$$

Here, $R_{\mathrm{c} \text {,bulk }}=\Delta L /\left(W C_{\mathrm{i}} \mu\left|V_{\mathrm{g}}-V_{\text {th }}-\frac{V_{\mathrm{d}}}{2}\right|\right)$ and $R_{\text {channel }}=L /\left(W C_{\mathrm{i}} \mu\left|V_{\mathrm{g}}-V_{\text {th }}-\frac{V_{\mathrm{d}}}{2}\right|\right)$. The values of $\Delta L$ do not affect the extracted $R_{\mathrm{c}, \text { int }}$. Then the values of $R_{\mathrm{c}, \text { int }}$ are set in the following forms: $R_{0} \exp \left(\frac{V_{0}-V_{\mathrm{d}}}{P_{1}}\right)$ (referred to as “Exp-1"), $\left(V_{\mathrm{d}}\right)^{P_{2}} \exp \left[-\left(V_{\mathrm{d}}\right)^{P_{2}}\right]$ (“Exp-2”), $R_{0}\left(V_{\mathrm{d}}\right)^{-P_{3}}$ (“Power"), $P_{4}$ (“Const"). In the calculation and simulation, $V_{\mathrm{d}}$ ranges from $0 \mathrm{~V}$ to $-30 \mathrm{~V}$ with a step of $-1 \mathrm{~V}$. In Fig. 2, the set values of $R_{\mathrm{c} \text {,int }}$ and calculated $R_{\mathrm{c} \text {,int }}$ extracted by Table 1 are compared. The values of calculation and simulation parameters can be found in SI, Part 2 .

Calculation and simulation of surface recombination rate. The surface recombination rate $r_{\text {rec }}$ can be calculated as the product of the surface charge density $n$ and the surface recombination velocity $S$. The voltage 
across the interface $V_{\mathrm{a}}$ and local electric field $\epsilon$ at the interface and are affected by $V_{\mathrm{d}}$. Assume the former is $V_{\mathrm{a}}=c_{1}\left(V_{\mathrm{d}}\right)^{\alpha}$ and the latter is $\epsilon=c_{2}\left(V_{\mathrm{d}}\right)^{\beta}$, and charge mobility near the interface follows the field-effect mobility $\mu_{\text {int }}=c_{3} \mu^{\gamma}$. Then $r_{\text {rec }}$ can be expressed by (unit in $\mathrm{cm}^{-2} \mathrm{~s}^{-1}$ ) (Fig. 7)

$$
r_{\text {rec }}\left(V_{\mathrm{d}}\right)=r_{\text {rec }, 0} \exp \left(\sqrt{\frac{q r_{\mathrm{C}} c_{2}}{k T}\left(V_{\mathrm{d}}\right)^{\beta}}\right)\left[1-\psi^{2} \frac{q r_{\mathrm{C}} c_{2}}{k T}\left(V_{\mathrm{d}}\right)^{\beta}\right]
$$

Here $\psi$ is a weak function of $\epsilon$ (see SI for details). The detailed derivation and values of parameters in calculations and simulations can be found in SI, Part 5.

\section{References}

1. Natali, D. \& Caironi, M. Charge Injection in Solution-Processed Organic Field-Effect Transistors: Physics, Models and Characterization Methods. Adv. Mater. 24, 1357-1387 (2012).

2. Ante, F. et al. Contact Resistance and Megahertz Operation of Aggressively Scaled Organic Transistors. Small 8, 73-79 (2012).

3. Greiner, M. T., Chai, L., Helander, M. G., Tang, W. M. \& Lu, Z. H. Metal/Metal-Oxide Interfaces: How Metal Contacts Affect the Work Function and Band Structure of MoO3. Adv. Func. Mater. 23, 215-226 (2013).

4. Xu, Y. et al. Joule's law for organic transistors exploration: Case of contact resistance. Journal of Applied Physics 113, 064507-064507064505 (2013).

5. Gelinck, G., Heremans, P., Nomoto, K. \& Anthopoulos, T. D. Organic Transistors in Optical Displays and Microelectronic Applications. Adv. Mater. 22, 3778-3798 (2010).

6. Guo, Y. L., Yu, G. \& Liu, Y. Q. Functional Organic Field-Effect Transistors. Adv. Mater. 22, 4427-4447 (2010).

7. Dong, H. L., Jiang, L. \& Hu, W. P. Interface engineering for high-performance organic field-effect transistors. Physical Chemistry Chemical Physics 14, 14165-14180 (2012).

8. Zhou, Y. H. et al. A Universal Method to Produce Low-Work Function Electrodes for Organic Electronics. Science 336, 327-332 (2012).

9. Greiner, M. T. et al. Universal energy-level alignment of molecules on metal oxides. Nature Materials 11, 76-81 (2012).

10. Marinkovic, M., Belaineh, D., Wagner, V. \& Knipp, D. On the origin of contact resistances of organic thin film transistors. Adv Mater 24, 4005-4009 (2012).

11. Paasch, G. \& Scheinert, S. Space charge layers in organic field-effect transistors with Gaussian or exponential semiconductor density of states. Journal of Applied Physics 101 (2007).

12. Khim, D. et al. Control of Ambipolar and Unipolar Transport in Organic Transistors by Selective Inkjet-Printed Chemical Doping for High Performance Complementary Circuits. Advacned Functional Materials 24, 6252-6261 (2014).

13. Zhao, N. et al. Polaron Localization at Interfaces in High-Mobility Microcrystalline Conjugated Polymers. Adv. Mater. 21, 3759-3763 (2009).

14. Troisi, A. \& Orlandi, G. Charge-transport regime of crystalline organic semiconductors: Diffusion limited by thermal off-diagonal electronic disorder. Physical Review Letters 96, 086601 (2006).

15. Sze, S. M. Physics of Semiconductor Devices. 2nd Edition edn, (JOHN WILEY\&SONS, Inc., 1981).

16. Ng, T. N., Silveira, W. R. \& Marohn, J. A. Dependence of charge injection on temperature, electric field, and energetic disorder in an organic semiconductor. Physical Review Letters 98, 066101 (2007).

17. Emtage, P. \& O'Dwyer, J. Richardson-Schottky effect in insulators. Physical review letters 16, 356 (1966).

18. Scott, J. C. \& Malliaras, G. G. Charge injection and recombination at the metal-organic interface. Chem. Phys. Lett. 299, 115-119 (1999).

19. Shen, Y. L., Klein, M. W., Jacobs, D. B., Scott, J. C. \& Malliaras, G. G. Mobility-Dependent Charge Injection into an Organic Semiconductor. Physical Review Letters 86, 3867-3870 (2001).

20. Imakawa, M., Sawabe, K., Yomogida, Y., Iwasa, Y. \& Takenobu, T. Extraction of the contact resistance from the saturation region of rubrene single-crystal transistors. Appl. Phys. Lett. 99, 233301 (2011).

21. Liu, C. et al. Direct and quantitative understanding of the non-Ohmic contact resistance in organic and oxide thin-film transistors. Organic electronics 27, 253-258 (2015).

22. Liu, C. et al. Effect of Doping Concentration on Microstructure of Conjugated Polymers and Characteristics in N-Type Polymer Field-Effect Transistors. Adv. Func. Mater. 25, 758-767 (2015).

23. Long, D. X. et al. Solution processed vanadium pentoxide as charge injection layer in polymer field-effect transistor with Mo electrodes. Organic Electronics 17, 66-76 (2015).

24. Kim, N.-K. et al. Solution-Processed Barium Salts as Charge Injection Layers for High Performance N-Channel Organic Field-Effect Transistors. ACS applied materials \& interfaces 6, 9614-9621 (2014).

25. Minari, T. et al. Highly Enhanced Charge Injection in Thienoacene-Based Organic Field-Effect Transistors with Chemically Doped Contact. Appl. Phys. Lett. 100, 093303 (2012).

26. Khim, D. et al. Large Enhancement of Carrier Transport in Solution-Processed Field-Effect Transistors by Fluorinated Dielectric Engineering. Adv. Mater. 28, 518-526 (2016).

27. Van der Holst, J., Van Oost, F., Coehoorn, R. \& Bobbert, P. Electron-hole recombination in disordered organic semiconductors: Validity of the Langevin formula. Physical Review B 80, 235202 (2009).

28. Juška, G., Genevičius, K., Nekrašas, N., Sliaužys, G. \& Österbacka, R. Two dimensional Langevin recombination in regioregular poly (3-hexylthiophe ne). Appl. Phys. Lett. 95, 013303 (2009).

29. Kuik, M., Koster, L., Wetzelaer, G. \& Blom, P. Trap-assisted recombination in disordered organic semiconductors. Physical review letters 107, 256805 (2011).

30. Long, D. X., Xu, Y., Wei, H.-x., Liu, C. \& Noh, Y.-Y. Controlling charge injection properties in polymer field-effect transistors by incorporation of solution processed molybdenum trioxide. Physical Chemistry Chemical Physics 17, 20160-20167 (2015).

\section{Acknowledgements}

The authors gratefully acknowledge the financial support of the project from National Natural Science Foundation of China (61504173), Guangdong Provincial Department of Science and Technology (2015B090924001), and Shunde Government (Contract No. 20140401), China. This work was also supported by the Center for Advanced Soft Electronics (2013M3A6A5073183) funded by the Ministry of Science, ICT, \& Future Planning and a National Research Foundation of Korea (NRF) grant funded by the Korean Government (MSIP) (NRF2014R1A2A2A01007159). CL \& YYN thank to Nam-Koo Kim for supplying a few device results.

\section{Author Contributions}

C.L. and Y.Y.N. designed the research. C.L. performed theoretical calculations; C.L., G.H., Y.X., D.X.L., W.T.P., X.L. and T.M. performed experiments and analysed the results. C.L. and Y.Y.N. wrote the paper. 


\section{Additional Information}

Supplementary information accompanies this paper at http://www.nature.com/srep

Competing financial interests: The authors declare no competing financial interests.

How to cite this article: Liu, C. et al. Universal diffusion-limited injection and the hook effect in organic thinfilm transistors. Sci. Rep. 6, 29811; doi: 10.1038/srep29811 (2016).

(c) (i) This work is licensed under a Creative Commons Attribution 4.0 International License. The images or other third party material in this article are included in the article's Creative Commons license, unless indicated otherwise in the credit line; if the material is not included under the Creative Commons license, users will need to obtain permission from the license holder to reproduce the material. To view a copy of this license, visit http://creativecommons.org/licenses/by/4.0/ 